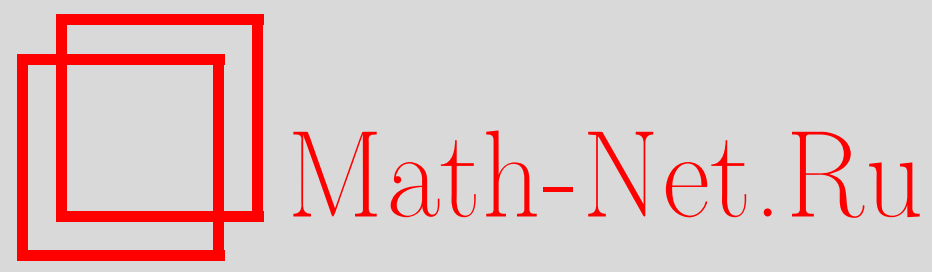

А. М. Попов, Обратная задача вариационного исчисления для систем дифференциально-разностных уравнений второго порядка, Матем. заметки, 2002, том 72, выпуск $5,745-749$

DOI: https://doi.org/10.4213/mzm464

Использование Общероссийского математического портала Math-Net.Ru подразумевает, что вы прочитали и согласны с пользовательским соглашением http://www . mathnet.ru/rus/agreement

Параметры загрузки:

IP : 3.85 .7 .115

26 апреля 2023 г., 16:43:07

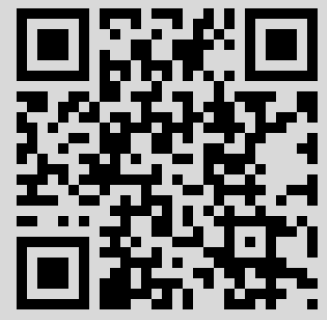




\section{ОБРАТНАЯ ЗАДАЧА ВАРИАЦИОННОГО ИСЧИСЛЕНИЯ ДЛЯ СИСТЕМ ДИФФЕРЕНЦИАЛЬНО-РАЗНОСТНЫХ УРАВНЕНИЙ ВТОРОГО ПОРЯДКА}

\section{А. М. Попов}

Рассмотрена обратная задача вариационного исчисления для нелинейной и линейной систем дифференциально-разностных уравнений второго порядка. Установлена связь между формальной потенциальностью линейной системы с постоянными коэффициентами и четностью ее характеристической функции.

Библиографиял: 4 названия.

Введение. Рассматривается классическая обратная задача вариационного исчисления [1, с. 12]. Пусть задан оператор $N$, определенный на множестве $D(N) \subset U$ и действующий в пространство $V(N: D(N) \rightarrow V)$. На множестве $V \times U$ задается симметричная билинейная форма $\Phi(v, g) \equiv\langle v, g\rangle$, отображающая множество $V \times U$ в множество действительных чисел $(\langle\cdot, \cdot\rangle: V \times U \rightarrow \mathbb{R})$. Требуется для оператора $N$ уравнения

$$
N(u)=O_{v}
$$

где $O_{v}$ - нулевой элемент пространства $V$, найти дифференцируемьй по Гато функционал $f[u]$ такой, что его вариация имеет вид

$$
\delta f[u]=\langle N(u), \delta u\rangle \quad \forall u \in D(N),
$$

т.е. уравнение (1) является уравнением Эйлера для функционала $f$.

Операторы, для которых существуют такие функционалы, называются потенииальными относительно заданной билинейной формы. При этом $f$ назьвается потенииалом оператора $N$.

Искомьй функционал для потенциального оператора $N$ находится по формуле [1, с. 15$]$

$$
f[u]=f\left[u_{0}\right]+\int_{0}^{1}\left\langle N\left(u_{0}+s\left(u-u_{0}\right)\right), u-u_{0}\right\rangle d s
$$

где $u_{0}-$ фиксированный элемент из $D(N)$.

Работа выполнена при финансовой поддержке Министерства образования РФ по программе "Университеты России - фундаментальные исследования", грант № 1199. 
Таким образом, чтобы построить искомьй функционал по заданному уравнению, вначале необходимо установить, является ли оператор уравнения потенциальньм. Для обыкновенных дифференциальных уравнений второго поря дка необходимые условия потенциальности соответствующих операторов впервые получены Гельмгольцем [2], поэтому в литературе подобные условия принято назьвать условиями потенциальности Гельмгольца.

В настоящей работе для систем дифференциально-разностых уравнений второго порядка осуществлена редукция условий потенциальности Гельмгольца, полученных в работе [3] для системы уравнений $\mu$-го порядка. Для линейной системы с потенциальным оператором построен потенциал. Установлена связь между формальной потенциальностью линейной системы с постоянными коэффициентами и четностью ее характеристической функции.

1. Нелинейная система. Рассмотрим систему дифференциально-разностых уравнений

$$
N_{i}(u) \equiv F_{i}\left(t, u(t+\theta \tau), u^{\prime}(t+\theta \tau), u^{\prime \prime}(t+\theta \tau)\right)=0
$$

где $i=1, \ldots, n, u(t)=\left(u_{1}(t), \ldots, u_{n}(t)\right)$ - неизвестная вектор-функция, $\theta=-1,0,1$, $\tau=$ const $>0, t \in\left[t_{1}-\tau, t_{2}+\tau\right], t_{2}-t_{1}>2 \tau, F_{i}$ - трижды непрерывно дифференцируемые функции по всем переменным в некоторой ограниченной области $\Omega \subset \mathbb{R}^{9 n+1}$.

Решением системы (3) будем назьвать непрерывную, дважды кусочно-дифференцируемую вектор-функцию, удовлетворяющую уравнению $(3)$ всюду на $\left[t_{1}-\tau, t_{2}+\tau\right]$, кроме конечного числа точек.

Обозначим через $U=Q^{2}$ множество непрерьвных дважды кусочно-дифференцируемых функций при $t \in\left[t_{1}-\tau, t_{2}+\tau\right]$. Зададим область определения оператора $N=$ $\left(N_{1}, N_{2}, \ldots, N_{n}\right)(3)$ равенством

$$
D(N)=\left\{u \in U: u(t)=\varphi_{j}(t), t \in E_{j}=\left[t_{j}-\tau, t_{j}+\tau\right], j=1,2\right\}
$$

где $\varphi_{j}(t)$ - заданные функции, $\varphi_{j}(t) \in C^{2}$.

Пусть $N: D(N) \rightarrow V$. На множестве $V \times U$ зададим локальную билинейную форму $\langle\cdot, \cdot\rangle: V \times U \rightarrow \mathbb{R}$ равенством

$$
\langle v, g\rangle=\int_{t_{1}}^{t_{2}} v(t) g(t) d t
$$

где

$$
v(t) g(t)=\sum_{k=1}^{n} v_{k}(t) g_{k}(t)
$$

ТЕОРема 1. Для того чтобы оператор $N$ системы уравнений (3) был потенциальным на заданном множсестве $D(N)$ (4) относительно билинейной формы (5), 
необходимо и достаточно, чтобы для любого $u \in D(N)$ выполнялись условия

$$
\begin{aligned}
{\left[-\frac{\partial F_{i}}{\partial u_{k}^{\prime}(t-\tau)}+2 \frac{d}{d t} \frac{\partial F_{i}}{\partial u_{k}^{\prime \prime}(t-\tau)}\right]_{+} } & =\frac{\partial F_{k}}{\partial u_{i}^{\prime}(t+\tau)}, \quad\left[\frac{\partial F_{i}}{\partial u_{k}^{\prime \prime}(t-\tau)}\right]_{+}=\frac{\partial F_{k}}{\partial u_{i}^{\prime \prime}(t+\tau)}, \\
\frac{\partial F_{i}}{\partial u_{k}^{\prime}(t)}+2 \frac{d}{d t} \frac{\partial F_{i}}{\partial u_{k}^{\prime \prime}(t)} & =\frac{\partial F_{k}}{\partial u_{i}^{\prime}(t)}, \quad \frac{\partial F_{i}}{\partial u_{k}^{\prime \prime}(t)}=\frac{\partial F_{k}}{\partial u_{i}^{\prime \prime}(t)} \\
\frac{\partial F_{k}}{\partial u_{i}(t+\tau)}-\left[\frac{\partial F_{i}}{\partial u_{k}(t-\tau)}\right]_{+} & =\frac{1}{2} \frac{d}{d t}\left(\frac{\partial F_{k}}{\partial u_{i}^{\prime}(t+\tau)}-\left[\frac{\partial F_{i}}{\partial u_{k}^{\prime}(t-\tau)}\right]_{+}\right) \\
\frac{\partial F_{k}}{\partial u_{i}(t)}-\frac{\partial F_{i}}{\partial u_{k}(t)} & =\frac{1}{2} \frac{d}{d t}\left(\frac{\partial F_{k}}{\partial u_{i}^{\prime}(t)}-\frac{\partial F_{i}}{\partial u_{k}^{\prime}(t)}\right)
\end{aligned}
$$

әде обозначено $[\Psi(t, u(t-\tau), u(t), \ldots)]_{+}=\Psi(t+\tau, u(t), u(t+\tau), \ldots),[\Psi]_{-}$определяется аналогично, $i, k=1, \ldots, n$.

Частные производные, входящие в равенства (6), находятся обычньп способом. Например, $\partial F_{i} / \partial u_{k}^{\prime}(t-\tau)$ означает частную производную по переменной $u_{k}^{\prime}(t-\tau)$.

ДокАЗАТЕЛЬСТво. Система уравнений (3) является частным случаем системы дифференциально-разностных уравнений $\mu$-го порядка, рассмотренной в работе [3], где для соответствующего оператора получены необходимые и достаточные условия потенциальности Гельмгольца относительно классической билиненой формы (5). Применительно к рассматриваемому оператору $N$ системы (3) эти условия имеют вид

$$
\begin{aligned}
& {\left[\frac{\partial F_{i}}{\partial u_{k}(t-\tau)}-\frac{d}{d t} \frac{\partial F_{i}}{\partial u_{k}^{\prime}(t-\tau)}+\frac{d^{2}}{d t^{2}} \frac{\partial F_{i}}{\partial u_{k}^{\prime \prime}(t-\tau)}\right]_{+}=\frac{\partial F_{k}}{\partial u_{i}(t+\tau)}} \\
& \frac{\partial F_{i}}{\partial u_{k}(t)}-\frac{d}{d t} \frac{\partial F_{i}}{\partial u_{k}^{\prime}(t)}+\frac{d^{2}}{d t^{2}} \frac{\partial F_{i}}{\partial u_{k}^{\prime \prime}(t)}=\frac{\partial F_{k}}{\partial u_{i}(t)} \\
& {\left[\frac{\partial F_{i}}{\partial u_{k}(t+\tau)}-\frac{d}{d t} \frac{\partial F_{i}}{\partial u_{k}^{\prime}(t+\tau)}+\frac{d^{2}}{d t^{2}} \frac{\partial F_{i}}{\partial u_{k}^{\prime \prime}(t+\tau)}\right]_{-}=\frac{\partial F_{k}}{\partial u_{i}(t-\tau)}} \\
& {\left[-\frac{\partial F_{i}}{\partial u_{k}^{\prime}(t-\tau)}+2 \frac{d}{d t} \frac{\partial F_{i}}{\partial u_{k}^{\prime \prime}(t-\tau)}\right]_{+}=\frac{\partial F_{k}}{\partial u_{i}^{\prime}(t+\tau)},} \\
& {\left[\frac{\partial F_{i}}{\partial u_{k}^{\prime \prime}(t-\tau)}\right]_{+}=\frac{\partial F_{k}}{\partial u_{i}^{\prime \prime}(t+\tau)}} \\
& -\frac{\partial F_{i}}{\partial u_{k}^{\prime}(t)}+2 \frac{d}{d t} \frac{\partial F_{i}}{\partial u_{k}^{\prime \prime}(t)}=\frac{\partial F_{k}}{\partial u_{i}^{\prime}(t)} \\
& \frac{\partial F_{i}}{\partial u_{k}^{\prime \prime}(t)}=\frac{\partial F_{k}}{\partial u_{i}^{\prime \prime}(t)}, \\
& {\left[-\frac{\partial F_{i}}{\partial u_{k}^{\prime}(t+\tau)}+2 \frac{d}{d t} \frac{\partial F_{i}}{\partial u_{k}^{\prime \prime}(t+\tau)}\right]_{-}=\frac{\partial F_{k}}{\partial u_{i}^{\prime \prime}(t-\tau)}} \\
& {\left[\frac{\partial F_{i}}{\partial u_{k}^{\prime \prime}(t+\tau)}\right]_{-}=\frac{\partial F_{k}}{\partial u_{i}^{\prime \prime}(t-\tau)} .}
\end{aligned}
$$

Анализ условий (7)-(15) показывает, что их число можно сократить. В силу очевидных равенств

$$
\left[[\Psi]_{-}\right]_{+}=\left[[\Psi]_{+}\right]_{-}=\Psi
$$


условия (11) и (15) эквиваленты. Используя равенства (11) и (16), легко доказать, что равенства (10) и (14) также эквивалентны. Установим эквивалентность равенств (7) и (9). Разрешая (10) относительно $\left[\frac{\partial}{\partial t} \frac{\partial F_{i}}{\partial u_{k}^{\prime \prime}(t-\tau)}\right]_{+}$и подставляя полученное выражение в (7), получим

$$
\frac{\partial F_{k}}{\partial u_{i}(t+\tau)}-\left[\frac{\partial F_{i}}{\partial u_{i}(t-\tau)}\right]_{+}=\frac{1}{2} \frac{d}{d t}\left(\frac{\partial F_{k}}{\partial u^{\prime}(t+\tau)}-\left[\frac{\partial F_{i}}{\partial u_{k}^{\prime}(t-\tau)}\right]_{+}\right) .
$$

Аналогично, используя (16), равенство (17) можно получить из равенств (9) и (14). Эквивалентность (7) и (9) установлена.

Равенство (8) с учетом (12) может быть также представлено в более симметричном виде

$$
\frac{\partial F_{k}}{\partial u_{i}(t)}-\frac{\partial F_{i}}{\partial u_{k}(t)}=\frac{1}{2} \frac{d}{d t}\left(\frac{\partial F_{k}}{\partial u_{i}^{\prime}(t)}-\frac{\partial F_{i}}{\partial u_{k}^{\prime}(t)}\right) .
$$

Отбрасьвая равенства (9), (14) и (15), как эквивалентные соответственно равенствам (7), (10) и (11), а условия (7) и (8) заменяя на более симметричные (17) и (18), получим, что необходимые и достаточные условия потенциальности оператора $N$ системы (3) могут быть представлены равенствами (10)-(13), (17) и (18), которые совпадают с условиями (6). Теорема доказана.

ЗАмЕчАнИЕ 1. Полученные условия потенциальности для рассматриваемого оператора также будем называть условиями потенциальности Гельмгольца.

ЗАмЕчАниЕ 2. Аналогичный результат для обыкновенных дифференциальных уравнений приведен в $[1$, с. 27 , теорема 2$]$.

2. Линейная система. Рассмотрим линейную систему дифференциально-разностных уравнений

$$
N(u) \equiv \sum_{l=0}^{2}\left(A_{l}(t) u^{(l)}(t-\tau)+B_{l}(t) u^{(l)}(t)+C_{l}(t) u^{(l)}(t+\tau)\right)+p(t)=O_{v}
$$

где $A_{l}, B_{l}, C_{l}$ - матрицы $(n \times n), p-n$-вектор, $A_{l}, B_{l}, C_{l}, p \in C^{2}, t \in\left[t_{1}-\tau, t_{2}+\tau\right]$.

ТЕОрема 2. Для того чтобы оператор $N$ системы уравнений (19) был потенииальным на множестве $D(N)$ (4) относительно билинейной формы (5), необходимо и достаточно, чтобы при $t \in[t-\tau, t+\tau]$ выполнялись условия

$$
\begin{gathered}
-A_{1}(t+\tau)+2 A_{2}^{\prime}(t+\tau)=C_{1}^{T}(t), \quad A_{2}(t+\tau)=C_{2}^{T}(t), \\
-B_{1}(t)+2 B_{2}^{\prime}(t)=B_{1}^{T}(t), \quad B_{2}(t)=B_{2}^{T}(t), \\
C_{0}^{T}(t)-A_{0}(t+\tau)=\frac{1}{2}\left(C_{1}^{\prime T}(t)-A_{1}^{\prime}(t+\tau)\right), \\
B_{0}^{T}(t)-B_{0}(t)=\frac{1}{2}\left(B_{1}^{\prime T}(t)-B_{1}^{\prime}(t)\right),
\end{gathered}
$$

где верхний индекс Т означает транспонирование матрицы.

Для доказательства теоремы достаточно применить условия потенциальности (6), установленные в теореме 1, к оператору $N(19)$. 
Пусть для оператора $N$ системы уравнений (19) выполняются условия потенциальности (20). Тогда его потенциал находится по формуле (2). Применяя интегрирование по частям слагаемых, содержащих вторые производные, а также замену переменной интегрирования в выражениях, содержащих аргумент $t+\tau$, найдем потенциал $f$ оператоpa $N(19)$ :

$$
\begin{aligned}
& f[u]=\frac{1}{2} \int_{t_{1}}^{t_{2}}\left(u^{T}(t)\left(A_{0}(t)+C_{0}^{T}(t-\tau)\right) u(t-\tau)+u^{T}(t)\left(A_{1}(t)-A_{2}^{\prime}(t)\right) u^{\prime}(t-\tau)\right. \\
& -2 u^{\prime T}(t) A_{2}(t) u^{\prime}(t-\tau)+u^{T}(t) B_{0}(t) u(t)+u^{T}(t)\left(B_{1}(t)-B_{2}^{\prime}(t)\right) u^{\prime}(t) \\
& \left.-u^{\prime T}(t) B_{2}(t) u^{\prime}(t)+u^{\prime T}(t)\left(C_{1}^{T}(t-\tau)-C_{2}^{\prime T}(t-\tau)\right) u(t-\tau)+u^{T}(t) p(t)\right) d t+\text { const. }
\end{aligned}
$$

3. Линейная система с постоянными коэффициентами. Рассмотрим линейную однородную систему

$$
N(u) \equiv \sum_{l=0}^{2}\left(A_{l} u^{(l)}(t-\tau)+B_{l} u^{(l)}(t)+C_{l} u^{(l)}(t+\tau)\right)=O_{v},
$$

где $A_{l}, B_{l}, C_{l}$ - постоянные матрицы $(n \times n)$.

ТЕОРема 3. Пусть для оператора $N$ системы (21) выполняются условия (20), т.е. имеют место равенства

$$
-A_{1}=C_{1}^{T}, \quad A_{2}=C_{2}^{T}, \quad B_{1}=0, \quad B_{2}=B_{2}^{T}, \quad C_{0}=A_{0}^{T}, \quad B_{0}=B_{0}^{T} .
$$

В этом случае будем говорить, что для системы (21) выполнены условия формальной потенциальности. Тогда характеристическая функция (квазиполином) системы (21) (см. [4, с. 427]) - четная функиия.

ДокАзАТЕЛЬСтво. Характеристическая функция $h(\lambda)$ системы $(21)$ имеет вид (см. $[4$, c. 427$])$

$$
h(\lambda)=\operatorname{det} H(\lambda)
$$

где

$$
H(\lambda)=\left(A_{0}+A_{1} \lambda+A_{2} \lambda^{2}\right) e^{-\lambda \tau}+B_{0}+B_{1} \lambda+B_{2} \lambda^{2}+\left(C_{0}+C_{1} \lambda+C_{2} \lambda^{2}\right) e^{\lambda \tau} .
$$

Очевидно, в силу равенств $(22) H(-\lambda)=H^{T}(\lambda)$. Поэтому

$$
h(-\lambda)=\operatorname{det} H(-\lambda)=\operatorname{det} H^{T}(\lambda)=h(\lambda) .
$$

Теорема доказана.

\section{СПИСОК ЦИТИРОВАННОЙ ЛИТЕРАТУРЫ}

[1] Филиппов В. М., Савчин В. М., Шорохов С. Г. Вариационные принципы для непотенциальных операторов // Итоги науки и техники. Современные проблемы математики. Новейшие достижения. Т. 40. М.: ВИНИТИ, 1992.

[2] Гельмгольц Г. О физическом значении принципа наименьшего действия // Вариационные принципы механики / ред. Л. С. Полак. М., 1959. С. 430-459.

[3] Попов А. М. Условия потенциальности Гельмгольца для систем дифференциально-разностных уравнений // Матем. заметки. 1998. Т. 64. № 3. С. 437-442.

[4] Беллман Р., Кук К. Дифференциально-разностные уравнения. М.: Мир, 1967. 